\title{
EXCITONIC MOLECULE IN CuCl CRYSTAL
}

\author{
W. UNGIER \\ Institute of Physics, Polish Academy of Sciences \\ Al. Lotników 32/46, 02-668 Warszawa, Poland \\ (Received February 1, 1993; in final form April 2, 1993)
}

\begin{abstract}
The electronic structure of a biexciton is discussed while taking into account the detailed band structure of $\mathrm{CuCl}$. The fine structure of the excitonic molecule is clarified with the effective electron-hole exchange effect taken into account beyond the effective mass approximation. The electron-hole exchange interaction mixes the states of opposite parities with respect to the permutation of electrons and holes. Two trial envelope functions, symmetric and antisymmetric under the permutation of two electrons or two holes, were used in the numerical minimization of the ground state energy of the biexciton. The obtained binding energy of the biexciton, as well as, the ratio of the mixing of the trial envelope functions of opposite parities are presented.
\end{abstract}

PACS numbers: $71.35 .+\mathrm{z}$

\section{Introduction}

The biexciton as the bound state of the two Wannier excitons is described in the effective mass approximation by the Hamiltonian, which differs from that of the $\mathrm{H}_{2}$ molecule, among other terms due to the effective electron-hole exchange. The electron-hole exchange interaction mixes states of opposite parity therefore the states of the biexciton have no defined parity under separate permutation of the spatial, as well as, the spin coordinates of identical particles, two electrons and two holes, respectively. It was shown by Ungier [1, 2] and Hayashi [3] in the previous papers that the ground state of the biexciton should be described by two-component spatial envelope function, symmetric and antisymmetric, but not by a symmetric wave function only, as in the case of hydrogen molecule. The previous papers refer to the simple band model neglecting the spin-orbit interaction in semiconductor. Here we consider the biexciton in real $\mathrm{CuCl}$ crystal. We consider the detailed band structure of $\mathrm{CuCl}$ calculated while taking into account the spin-orbit interaction.

For the optimization of the ground state energy of the biexciton we use the two-component trial envelope functions of the type used by Brinkman et al. [4]. 
We note that the analogous effect of mixing of the states of the opposite parities with respect to the electron-hole exchange takes place in the bound exciton complexes, i.e., in the case of exciton bound to neutral donor, where the symmetry under the permutation of two electrons is considered $[5,6]$.

\section{Two-band model of the crystal}

$\mathrm{CuCl}$, the crystal of symmetry $T_{d}$, has a band structure characterized with the top of the valence band at $k=0$ (symmetry $\Gamma_{7}$ ) and the bottom of the conduction band also at $k=0$ (symmetry $\Gamma_{6}$ ). The notation of Koster et al. [7] for the irreducible representations of space groups is used throughout this paper. The energy gap $E_{\mathrm{G}}\left(\Gamma_{6}^{c}-\Gamma_{7}^{v}\right)$ is $3.429 \mathrm{eV}$. A lower fourfold degenerate valence band of symmetry $\Gamma_{8}$ is split off by the spin-orbit interaction by about $69 \mathrm{meV}$.

The conduction Bloch functions of $\Gamma_{6}$ can be written as

$$
c_{1 / 2}=\phi_{c}|\uparrow\rangle \text { and } \quad c_{-1 / 2}=\phi_{c}|\downarrow\rangle \text {, }
$$

where $\phi_{c}$ can be identified with the $4 s$ functions of $\mathrm{Cu}$. The top valence Bloch functions of $\Gamma_{7}$ are of the form [8]

$$
v_{1 / 2}=\sqrt{\frac{2}{3}} \psi_{1}|\downarrow\rangle+\sqrt{\frac{1}{3}} \psi_{0}|\uparrow\rangle \text { and } v_{-1 / 2}=\sqrt{\frac{2}{3}} \psi_{-1}|\uparrow\rangle-\sqrt{\frac{1}{3}} \psi_{0}|\downarrow\rangle
$$

where $\psi_{ \pm 1}=(1 / \sqrt{2})\left(p_{x}+\mathrm{i} p_{y}\right)$ and $\psi_{0}=p_{z}$. The $p$-like functions $p_{x}, p_{y}, p_{z}$ may be identified with the $3 p$ functions of $\mathrm{Cl}$. The states $|\uparrow\rangle$ and ||$\rangle$ are the pure spin eigenstates of the spin operator of $s=1 / 2,|\uparrow\rangle$ according to the eigenvalue $+\hbar / 2$ and $|\downarrow\rangle$ to $-\hbar / 2$. In this approximation the $\Gamma_{7}$ states coincide with those of total angular momentum $J=1 / 2\left(m_{J}=-1 / 2\right.$ and $\left.m_{J}=+1 / 2\right)$.

We assume that the one-electron Bloch functions

$$
\psi_{n, k}=\frac{1}{\sqrt{N}} \mathrm{e}^{\mathrm{i} k \cdot r} u_{n, k}(r) \quad(n=c, v)
$$

( $k$ stands for $k, m_{J} ; u_{n, k}$ is a periodic function with the period of the crystal lattice and is normalized to unity in a unit cell of volume $\Omega, \psi_{n, k}$ is normalized in a large volume $V$ of $N=V / \Omega$ ) satisfy the usual Hartree-Fock equation

$$
H_{\mathrm{HF}} \psi_{n, k}=E_{n}(k) \psi_{n, k} .
$$

For the $2 N$-electron problem described by the Hamiltonian

$$
H=\sum_{i}^{2 N}\left\{-\frac{\hbar^{2}}{2 m} \Delta_{i}+V_{\text {per }}\left(r_{i}\right)\right\}+\frac{1}{2} \sum_{i \neq j} \frac{e^{2}}{r_{i j}},
$$

the ground state is given by a Slater determinant

$$
|\phi\rangle_{0}=((2 N) !)^{-1 / 2} \operatorname{det}\left\{\left|\psi_{v, k_{1}}(1) \psi_{v, k_{2}}(2) \ldots \psi_{v, k_{2 N}}(2 N)\right\rangle\right\} .
$$

Any excited states we will construct from $|\phi\rangle_{0}$ by substituting some valence wave functions $\left|\psi_{v, k}(i)\right\rangle$ with conduction states $\left|\psi_{c, l}(i)\right\rangle$ in Eq. (6). 


\section{Bound states of the biexciton}

The biexciton wave function is defined as a linear combination

$$
|\phi\rangle=\sum_{\substack{l l^{\prime} \\
k k^{\prime}}} B_{K}\left(\begin{array}{c}
1 l^{\prime} \\
k k^{\prime}
\end{array}\right)\left|\phi_{k}^{l} l_{k \prime}^{\prime}\right\rangle
$$

where $K$ labels the total momentum wave vector of the state and $\left|\phi_{k k^{\prime}}^{l} l^{\prime}\right\rangle$ denotes a Slater determinant in which two-valence wave functions $\psi_{v, k}$ and $\psi_{v, k}$ in Eq. (6) are substituted by conduction wave functions $\psi_{c, l}$ and $\psi_{c, l}$, respectively.

The stationary Schrödinger equation

$$
H|\phi\rangle=E_{(\text {biex })}|\phi\rangle
$$

determines the expansion coefficients $B_{K}\left(\begin{array}{l}1 \\ k \\ k k^{\prime}\end{array}\right)$ in Eq. (7) and the biexciton energies.

We consider the biexciton formed from shallow Wannier excitons and we make the assumption that the periodic parts of the Bloch functions, $u_{n, k}$, vary slowly with $k$ over the range of interest - that is over the range of $l, l^{\prime}, k, k^{\prime}$ for which $B_{K}\left(\begin{array}{l}l l^{\prime} \\ k k^{\prime}\end{array}\right)$ is appreciable - and that this range is small compared to the dimensions of the Brillouin zone. The coefficients $B_{K}\left(\begin{array}{l}l \\ k k^{\prime}\end{array}\right)$ are non-vanishing for $l+l^{\prime}-k-k^{\prime}=K$ only.

The restriction to the subspace of the $\left|\phi_{k}^{l} z_{k^{\prime}}\right\rangle$ allows one to reduce formally the $2 N$-electron biexciton problem to a few particle problem by associating to each function $\left|\phi_{k}^{l} l_{k^{\prime}}^{l^{\prime}}\right\rangle$ the two-electron and two-hole function

$$
\begin{aligned}
\left|\bar{\phi}_{k k^{\prime}}^{l l^{\prime}}\right\rangle & =\frac{1}{2}\left[\psi_{c, l}\left(r_{1}\right) \psi_{c, l^{\prime}}\left(r_{3}\right)-\psi_{c, l^{\prime}}\left(r_{1}\right) \psi_{c, l}\left(r_{3}\right)\right] \\
& \times K(2) K(4)\left[\psi_{v, k}\left(r_{2}\right) \psi_{v, k^{\prime}}\left(r_{4}\right)-\psi_{v, k^{\prime}}\left(r_{2}\right) \psi_{v, k}\left(r_{4}\right)\right],
\end{aligned}
$$

where $K=-\mathrm{i} \sigma_{y} K_{0}$ is the usual time reversal operator and $K \psi_{v, k}$ is the state of the hole. Indexes 1,3 refer to two electrons, indexes 2,4 - to two holes.

In the subspace spanned by $\left|\bar{\phi}_{k k^{\prime}}^{l l^{\prime}}\right\rangle$ the $2 N$-electron Hamiltonian $H$ is replaced by $H^{\text {(biex) }}$, defined by the equation

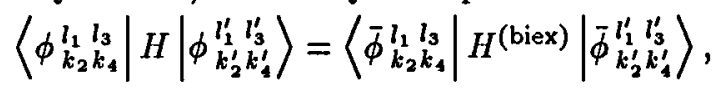

which should be satisfied for all $l, l^{\prime}, k, k^{\prime}$. It was shown [9] that $H^{(\text {biex) }}$ can be defined as

$$
\begin{aligned}
H^{(\text {biex })} & =E_{0}+\sum_{i=1,3} H_{\mathrm{HF}}(i)-\sum_{j=2,4} K(j) H_{\mathrm{HF}}(j) K^{\dagger}(j) \\
& +\frac{e^{2}}{\epsilon}\left(\frac{1}{r_{13}}+\frac{1}{r_{24}}-\sum_{\substack{i=1,3 \\
j=2,4}} \frac{1}{r_{i j}}\right)+\sum_{\substack{i=1,3 \\
j=2,4}} W(i, j),
\end{aligned}
$$

where $E_{0}$ is the ground state energy of the electrons filling the valence band, $e^{2} / \epsilon r_{i j}$ are the screened Coulomb interaction terms $\left(r_{i j}\right.$ is the interparticle distance, $\epsilon$ is the static dielectric constant). The effective electron-hole exchange term

$$
W(i, j)=V\left(r_{i}, r_{j}\right) V_{\mathrm{spin}}(i, j)
$$


acting on the functions with separate pure spin part $\psi_{n, k}|s\rangle$ (with $|s\rangle=|\uparrow\rangle$ or $|s\rangle=|\downarrow\rangle)$, reads as a projection operator

$$
V_{\text {spin }}(i, j)=\left(\left|\uparrow_{i}\right\rangle\left|\downarrow_{j}\right\rangle-\left|\downarrow_{i}\right\rangle\left|\uparrow_{j}\right\rangle\right)\left(\left\langle\uparrow_{i}\right|\left\langle\downarrow_{j}\right|-\left\langle\downarrow_{i}\right|\left\langle\uparrow_{j}\right|\right)
$$

and

$$
\begin{aligned}
& \left\langle\psi_{c, l}\left(r_{i}\right) \psi_{v, k}\left(r_{j}\right)\left|V\left(r_{i}, r_{j}\right)\right| \psi_{c, l^{\prime}}\left(r_{i}\right) \psi_{v, k^{\prime}}\left(r_{j}\right)\right\rangle \\
& =\iint \mathrm{d}^{3} r_{i} \mathrm{~d}^{3} r_{j}\left(\psi_{c, l}\left(r_{i}\right) \psi_{v, k}\left(r_{i}\right)\right)^{*} \frac{e^{2}}{r_{i j}} \psi_{c, l^{\prime}}\left(r_{j}\right) \psi_{v, k^{\prime}}\left(r_{j}\right) .
\end{aligned}
$$

For the four-particle space of the biexciton we have sixteen basis functions. Since the conduction and valence basis states (1), (2) are the eigenstates of the one-electron total angular momentum operators of $J=1 / 2$, the four-particle states are the eigenstates of the four-particle total angular momentum operators of $J=0,1,2$. The crystal symmetries of the biexciton states are given by the following products of irreducible representations:

$$
\Gamma_{6} \otimes \Gamma_{7}^{*} \otimes \Gamma_{6} \otimes \Gamma_{7}^{*}=\left(\Gamma_{2}+\Gamma_{5}\right) \otimes\left(\Gamma_{2}+\Gamma_{5}\right)=2 \Gamma_{1}+3 \Gamma_{4}+\Gamma_{3}+\Gamma_{5} .
$$

In the total momentum classification $\Gamma_{1}$ corresponds to $J=0, \Gamma_{4}$ to $J=1$ and $\Gamma_{3}+\Gamma_{5}$ results from the splitting of $J=2$. We can attribute each of the four-particle basis states to one of the irreducible representation $\Gamma_{i}$ of $T_{d}$. Each of these sixteen states $\left|\Gamma_{i} \mu \nu\right\rangle$ can be chosen with definite permutational parity the symbol $\mu(\nu)$ stands for the sign + or - , respectively, in order to indicate the parity of the spin function under the permutation of the two electrons (holes). Only appropriate combinations of the two-electron and two-hole singlets and triplets are the eigenstates of the total (four-particle) angular momentum operators $\mathrm{J}^{2}$ and $J_{z},|\mu \nu ; J, m\rangle$. As a consequence there are possible only some parities of the $\left|\Gamma_{i} \mu \nu\right\rangle$ states. Denoting by $|0,0\rangle_{\mathrm{e}}$ the two-electron singlet $(\mu=-1)$

$$
|0,0\rangle_{\mathrm{e}}=\frac{1}{\sqrt{2}}\left[\left|c_{1 / 2}(1)\right\rangle\left|c_{-1 / 2}(3)\right\rangle-\left|c_{-1 / 2}(1)\right\rangle\left|c_{1 / 2}(3)\right\rangle\right]
$$

and by $|1, m\rangle_{\mathrm{e}}$ the two-electron triplet $(\mu=+1)$

$$
|1, m\rangle_{e}= \begin{cases}\left|c_{1 / 2}(1)\right\rangle\left|c_{1 / 2}(3)\right\rangle & \text { for } m=+1 \\ \frac{1}{\sqrt{2}}\left[\left|c_{1 / 2}(1)\right\rangle\left|c_{-1 / 2}(3)\right\rangle+\left|c_{-1 / 2}(1)\right\rangle\left|c_{1 / 2}(3)\right\rangle\right] & \text { for } m=0 \\ \left|c_{-1 / 2}(1)\right\rangle\left|c_{-1 / 2}(3)\right\rangle & \text { for } m=-1\end{cases}
$$

and by $|0,0\rangle_{\mathrm{h}}$ and $|1, m\rangle_{\mathrm{h}}$ the two-hole singlet $(\nu=-1)$

$$
|0,0\rangle_{\mathrm{h}}=\frac{1}{\sqrt{2}}\left[\left|v_{1 / 2}(2)\right\rangle\left|v_{-1 / 2}(4)\right\rangle-\left|v_{-1 / 2}(2)\right\rangle\left|v_{1 / 2}(4)\right\rangle\right]
$$

and triplet $(\nu=+1)$

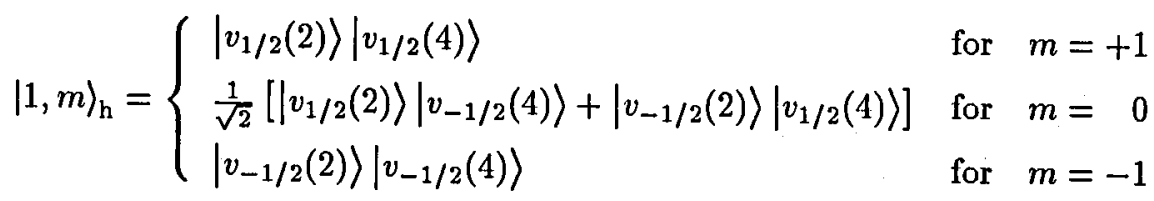


we may express the $\left|\Gamma_{i} \mu \nu\right\rangle$ states as follows:

$$
\begin{aligned}
&\left|\Gamma_{1}--\right\rangle=|0,0\rangle_{\mathrm{e}}|0,0\rangle_{\mathrm{h}} \\
&\left|\Gamma_{1}++\right\rangle= \frac{1}{\sqrt{3}}\left[|1,1\rangle_{\mathrm{e}}|1,-1\rangle_{\mathrm{h}}-|1,0\rangle_{\mathrm{e}}|1,0\rangle_{\mathrm{h}}+|1,-1\rangle_{\mathrm{e}}|1,1\rangle_{\mathrm{h}}\right] \\
&\left|\Gamma_{4}-+\right\rangle=|0,0\rangle_{\mathrm{e}} \times \begin{cases}|1,1\rangle_{\mathrm{h}} \\
|1,0\rangle_{\mathrm{h}} & \left|\Gamma_{4}+-\right\rangle=|0,0\rangle_{\mathrm{h}} \times\left\{\begin{array}{l}
|1,1\rangle_{\mathrm{e}} \\
|1,0\rangle_{\mathrm{e}}
\end{array}\right. \\
|1,-1\rangle_{\mathrm{h}}\end{cases} \\
&\left|\Gamma_{4}++\right\rangle=\left\{\begin{array}{l}
\frac{1}{\sqrt{2}}\left[|1,0\rangle_{\mathrm{e}}|1,1\rangle_{\mathrm{h}}-|1,1\rangle_{\mathrm{e}}|1,0\rangle_{\mathrm{h}}\right] \\
\frac{1}{\sqrt{2}}\left[|1,-1\rangle_{\mathrm{e}}|1,1\rangle_{\mathrm{h}}-|1,1\rangle_{\mathrm{e}}|1,-1\rangle_{\mathrm{h}}\right]
\end{array}\right. \\
&\left|\Gamma_{3}++\right\rangle=\left\{\begin{array}{l}
\frac{1}{\sqrt{6}}\left[|1,0\rangle_{\mathrm{e}}|1,-1\rangle_{\mathrm{h}}-|1,-1\rangle_{\mathrm{e}}|1,0\rangle_{\mathrm{h}}\right] \\
\frac{1}{\sqrt{2}}\left[|1,1\rangle_{\mathrm{e}}|1,1\rangle_{\mathrm{h}}+|1,-1\rangle_{\mathrm{e}}|1,-1\rangle_{\mathrm{h}}\right]
\end{array}\right. \\
&\left|\Gamma_{5}++\right\rangle=\left\{\begin{array}{l}
\frac{i}{2}\left[|1,0\rangle_{\mathrm{e}}\left(|1,1\rangle_{\mathrm{h}}+|1,-1\rangle_{\mathrm{h}}\right)+|1,0\rangle_{\mathrm{h}}\left(|1,1\rangle_{\mathrm{e}}+|1,-1\rangle_{\mathrm{e}}\right)\right] \\
-\frac{1}{2}\left[|1,0\rangle_{\mathrm{e}}\left(|1,1\rangle_{\mathrm{h}}-|1,-1\rangle_{\mathrm{h}}\right)+|1,0\rangle_{\mathrm{h}}\left(|1,1\rangle_{\mathrm{e}}-|1,-1\rangle_{\mathrm{e}}\right)\right] \\
-\frac{\mathrm{i}}{\sqrt{2}}\left[|1,1\rangle_{\mathrm{e}}|1,1\rangle_{\mathrm{h}}-|1,-1\rangle_{\mathrm{e}}|1,-1\rangle_{\mathrm{h}}\right]
\end{array}\right.
\end{aligned}
$$

In order to ensure the proper symmetry of the wave function of identical fermions, each $\left|\Gamma_{i} \mu \nu\right\rangle$ of definite permutational parity has to be multiplied by the spatial function of opposite parity

$$
\begin{aligned}
& \left|\bar{\mu} \bar{\nu} \begin{array}{ll}
l_{1} & l_{3} \\
k_{2} & k_{4}
\end{array}\right\rangle=\frac{1}{2}\left(\mathrm{e}^{\mathrm{i} l_{1} \cdot r_{1}+\mathrm{i} l_{3} \cdot r_{3}}+\bar{\mu} \mathrm{e}^{\mathrm{i} l_{3} \cdot r_{1}+\mathrm{i} l_{1} \cdot r_{3}}\right) \\
& \times\left(\mathrm{e}^{-\mathrm{i} \boldsymbol{k}_{2} \cdot \boldsymbol{r}_{2}-\mathrm{i} \boldsymbol{k}_{4} \cdot \boldsymbol{r}_{4}}+\bar{\nu} \mathrm{e}^{-\mathrm{i} \boldsymbol{k}_{4} \cdot \boldsymbol{r}_{2}-\mathrm{i} \boldsymbol{k}_{2} \cdot \boldsymbol{r}_{4}}\right),
\end{aligned}
$$

where $\bar{\mu}=-\mu$ and $\bar{\nu}=-\nu$.

Now we consider the stationary Schrödinger equation (8)

$$
H^{\text {(biex) }}|\bar{\phi}\rangle=E_{\text {(biex) }}|\bar{\phi}\rangle,
$$

for the biexciton wave function $|\bar{\phi}\rangle$ expanded in terms of the four-particle states

$$
|\rangle=\left|\bar{\mu} \bar{\nu} \begin{array}{cc}
l_{1} & l_{3} \\
k_{2} & k_{4}
\end{array}\right\rangle\left|\Gamma_{i} \mu \nu\right\rangle
$$

In the previous treatments $[8,9]$ it was always assumed that each biexciton eigenstate $|\bar{\phi}\rangle$ is proportional to the one of $\left|\Gamma_{i} \mu \nu\right\rangle$ of definite parity under the permutation of two electrons and two holes. However, the states $\left|\Gamma_{1}-\right\rangle$ and $\left|\Gamma_{1}++\right\rangle$ are mixed by the effective electron-hole exchange interaction (see Appendix), and they are not mixed with any other $\left|\Gamma_{i} \mu \nu\right\rangle$ states. Taking this mixing into consideration we define the biexciton $\Gamma_{1}$ state 


$$
\begin{aligned}
|\bar{\phi}\rangle= & \sum_{\substack{l_{1} l_{3} \\
k_{2} k_{4}}} B_{K}\left(\begin{array}{l}
l_{1} l_{3} \\
k_{2} k_{4}
\end{array}\right)\left|\bar{\phi}_{k_{2} k_{4}}^{l_{1} l_{3}}\right\rangle=2 \sum_{\substack{l_{1} l_{3} \\
k_{2} k_{4}}} B_{K}\left(\begin{array}{l}
l_{1} l_{3} \\
k_{2} k_{4}
\end{array}\right) \mathrm{e}^{\mathrm{i} l_{1} \cdot r_{1}} c_{m_{1}}(1) \\
& \times \mathrm{e}^{\mathrm{i} l_{3} \cdot r_{3}} c_{m_{3}}(3) K(2)\left(\mathrm{e}^{\mathrm{i} k_{2} \cdot r_{2}} v_{m_{2}}(2)\right) K(4)\left(\mathrm{e}^{\mathrm{i} k_{4} \cdot r_{4}} v_{m_{4}}(4)\right)
\end{aligned}
$$

$\left(l_{i} \equiv\left(l_{i}, m_{i}\right), k_{j} \equiv\left(k_{j}, m_{j}\right)\right)$, as a linear combination of the states $\left|\Gamma_{1}-\right\rangle$ and $\left.\mid \Gamma_{1}++\right)$

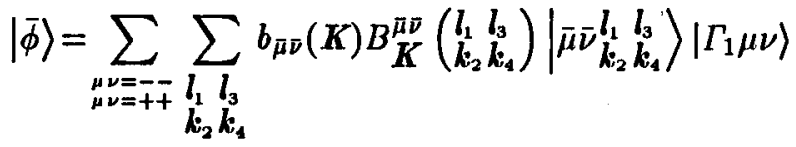

$$
\begin{aligned}
& =2 \sum_{\substack{l_{1} l_{3} \\
k_{2} k_{1}}} \exp \left[\mathrm{i}\left(\boldsymbol{l}_{1} \cdot \boldsymbol{r}_{1}+\boldsymbol{l}_{3} \cdot \boldsymbol{r}_{3}-\boldsymbol{k}_{2} \cdot \boldsymbol{r}_{2}-\boldsymbol{k}_{4} \cdot \boldsymbol{r}_{4}\right)\right] \\
& \times\left(b_{++}(K) B_{K}^{++}\left(\begin{array}{ll}
l_{1} & l_{3} \\
k_{2} & k_{4}
\end{array}\right)\left|\Gamma_{1}--\right\rangle+b_{--}(K) B_{K}^{-}\left(\begin{array}{ll}
l_{1} & l_{3} \\
k_{2} & k_{4}
\end{array}\right)\left|\Gamma_{1}++\right\rangle\right) .
\end{aligned}
$$

Since electrons and holes are fermions, the following antisymmetric relations have been assumed:

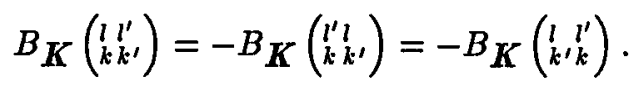

In Eq. (25) $\bar{\mu}, \bar{\nu}$ indicate the symmetry or antisymmetry of the cocflicients

$$
B_{K}^{\bar{\mu} \bar{\nu}}\left(\begin{array}{l}
l \\
l l^{\prime} \\
k
\end{array}\right)=\bar{\mu} B_{K}^{\bar{\mu} \bar{\nu}}\left(\begin{array}{l}
l^{\prime} l \\
k \\
k^{\prime}
\end{array}\right)=\bar{\nu} B_{K}^{\bar{\mu} \bar{\nu}}\left(\begin{array}{ll}
l & l^{\prime} \\
k^{\prime} k
\end{array}\right) \text {. }
$$

By the identification of Eq. (24) and Eq. (25) we find the explicit relations between the coefficients $B_{K}\left(\begin{array}{l}l \\ k k^{\prime}\end{array}\right)$ and $B_{K}^{\bar{\mu} \bar{\nu}}\left(\begin{array}{l}l \\ l \\ k k^{\prime}\end{array}\right)$ :

$$
\begin{aligned}
& B_{K}\left(\begin{array}{l}
l_{1} l_{3} \\
k_{2} k_{4}
\end{array}\right)=b_{++}(K) B_{K}^{++}\left(\begin{array}{ll}
l_{1} l_{3} \\
k_{2} k_{4}
\end{array}\right) \\
& \quad \times \frac{1}{2}\left(\delta_{m_{1}, \frac{1}{2}} \delta_{m_{3},-\frac{1}{2}}-\delta_{m_{3}, \frac{1}{2}} \delta_{m_{1},-\frac{1}{2}}\right)\left(\delta_{m_{2}, \frac{1}{2}} \delta_{m_{4},-\frac{1}{2}}-\delta_{m_{4}, \frac{1}{2}} \delta_{m_{2},-\frac{1}{2}}\right) \\
& +b_{--}(K) B_{K}^{--}\left(\begin{array}{ll}
l_{1} & l_{3} \\
k_{2} k_{4}
\end{array}\right) \\
& \quad \times \frac{1}{\sqrt{3}}\left[\delta_{m_{1, \frac{1}{2}}} \delta_{m_{3, \frac{1}{2}}} \delta_{m_{2}, \frac{1}{2}} \delta_{m_{1}, \frac{1}{2}}+\delta_{m_{1},-\frac{1}{2}} \delta_{m_{3},-\frac{1}{2}} \delta_{m_{2},-\frac{1}{2}} \delta_{m_{4},-\frac{1}{2}}\right. \\
& \left.\quad+\frac{1}{2}\left(\delta_{m_{1}, \frac{1}{2}} \delta_{m_{3},-\frac{1}{2}}+\delta_{m_{3}, \frac{1}{2}} \delta_{m_{1},-\frac{1}{2}}\right)\left(\delta_{m_{2}, \frac{1}{2}} \delta_{m_{4},-\frac{1}{2}}+\delta_{m_{4}, \frac{1}{2}} \delta_{m_{2},-\frac{1}{2}}\right)\right],
\end{aligned}
$$

where the relation $K \psi_{v, k, m_{J}}=(-1)^{J+m_{J}} \psi_{v,-k,-m_{J}}$, i.e. $K v_{1 / 2}=-v_{-1 / 2}$, $K v_{-1 / 2}=v_{1 / 2}$, has been taken into account.

We multiply the Schrödinger equation (22) by the bra \langle|$=\left\langle\Gamma_{1} \mu \nu\right|\left\langle\bar{\mu} \bar{\nu} \begin{array}{ll}l_{1} & l_{3} \\ k_{2} & k_{1}\end{array}\right|$ and we get for the $\Gamma_{1}$ state of the biexciton the equation

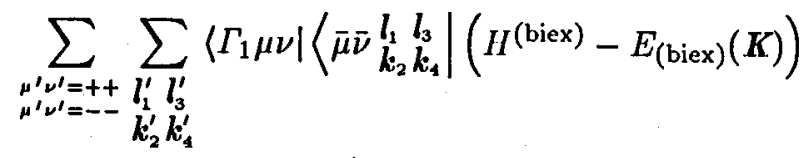

$$
\begin{aligned}
& \left.\times \mid \bar{\mu}^{\prime} \bar{\nu}^{\prime} \begin{array}{l}
l_{1}^{\prime} l_{3}^{\prime} \\
k_{2}^{\prime} k_{4}^{\prime}
\end{array}\right)\left|\Gamma_{1} \mu^{\prime} \nu^{\prime}\right\rangle b_{\bar{\mu}^{\prime} \bar{\nu}^{\prime}}(K) B_{K}^{\bar{\mu}^{\prime} \bar{\nu}^{\prime}}\left(\begin{array}{l}
l_{1}^{\prime} \\
l_{3}^{\prime} \\
k_{2}^{\prime} k_{4}^{\prime}
\end{array}\right)=0 .
\end{aligned}
$$


Similarly to the exciton problem we introduce the Fourier transforms of the coefficients $B_{K}^{\not \mu \nu}[10]$

$$
\begin{aligned}
& \Phi_{K}^{\bar{\mu} \bar{\nu}}\left(r_{1}, r_{3}, r_{2}, r_{4}\right)=\frac{1}{V^{2}} \sum_{\substack{l_{1} l_{3} \\
k_{2} k_{4}}} B_{K}^{\bar{\mu} \bar{\nu}}\left(\begin{array}{ll}
l_{1} & l_{3} \\
k_{2} & k_{4}
\end{array}\right) \\
& \quad \times \exp \left[\mathrm{i}\left(l_{1} \cdot r_{1}+l_{3} \cdot r_{3}-k_{2} \cdot r_{2}-k_{4} \cdot r_{4}\right)\right],
\end{aligned}
$$

which satisfy the symmetry relations

$$
\Phi_{K}^{\overline{\mu \nu}}\left(r_{1}, r_{3}, r_{2}, r_{4}\right)=\bar{\mu} \Phi_{K}^{\bar{\mu} \bar{\nu}}\left(r_{3}, r_{1}, r_{2}, r_{4}\right)=\bar{\nu} \Phi_{K}^{\mu \nu}\left(r_{1}, r_{3}, r_{4}, r_{2}\right)
$$

Taking the Fourier transform of Eq. (29) we sliow in Appendix that the functions $\Phi_{K}^{\bar{\mu} \bar{\nu}}$ satisfy the matrix-differential effective mass equation

$$
\left[\begin{array}{cr}
H_{0}+\frac{1}{2} \tilde{J} \delta_{+}-E_{(\text {biex })}(\boldsymbol{K}) ; & -\frac{1}{2 \sqrt{3}} \tilde{J} \delta_{-} \\
-\frac{1}{2 \sqrt{3}} \tilde{J} \delta_{-} ; & H_{0}+\frac{1}{6} \tilde{J} \delta_{+}-E_{(\text {biex })}(K)
\end{array}\right]\left[\begin{array}{c}
b_{++}(K) \Phi_{K}^{++} \\
b_{--}(K) \Phi_{K}^{--}
\end{array}\right]=0
$$

where

$$
\begin{aligned}
I_{0}= & E_{0}+E_{c}\left(-\mathrm{i} \nabla_{1}\right)+E_{c}\left(-\mathrm{i} \nabla_{3}\right)-E_{v}\left(-\mathrm{i} \nabla_{2}\right)-E_{v}\left(-\mathrm{i} \nabla_{4}\right) \\
& +\frac{e^{2}}{c}\left(\frac{1}{r_{13}}+\frac{1}{r_{24}}-\frac{1}{r_{12}}-\frac{1}{r_{14}}-\frac{1}{r_{32}}-\frac{1}{r_{34}}\right)
\end{aligned}
$$

represents the kinetic and Coulomb energy of the four particles, (the vectors $\boldsymbol{l}_{i}, \boldsymbol{k}_{j}$ have been replaced with the operators $-\mathrm{i} \nabla_{i},-\mathrm{i} \nabla_{j}$ in the matrix elements of the onc-particle IIamiltonians contained in $H^{(\text {biex })}(11)$ ),

$$
\delta_{ \pm}=\Omega\left[\delta\left(r_{1}-r_{2}\right) \pm \delta\left(r_{1}-r_{4}\right)+\delta\left(r_{3}-r_{4}\right) \pm \delta\left(r_{3}-r_{2}\right)\right]
$$

and $\tilde{J}$ is the exchange integral for transverse excitons defined in Appendix (A8). We adopt Dirac $\delta$-functions since the exchange interaction is assumed to be a short-range one effective only in $\Omega$ - the volume of the unit cell.

For the normalized functions $\Phi_{K}^{\bar{\mu} \bar{\nu}}$ the coefficients $b_{\bar{\mu} \bar{\nu}}(K)$ satisfy the equation

$$
\left|b_{++}(K)\right|^{2}+\left|b_{--}(K)\right|^{2}=1 .
$$

For each vector $K$ there are two solutions for the set $\left(b_{++}(K), b_{--}(K)\right)$. For the small biexciton kinetic energies $(K \approx 0)$ one of the solution, that with $\left|b_{++}(K)\right| \approx 1$ corresponds to the bound state. The next higher biexciton state of the symmetry $\Gamma_{4}$ is also bound in $\mathrm{CuCl}[8]$ because of the very small electron-hole mass ratio $m_{\mathrm{e}} / m_{\mathrm{h}}=0.02$. Similarly, as in the case of $\Gamma_{1}$ states, the states $\left|\Gamma_{4}-+\right\rangle$, $\left|\Gamma_{4}+-\right\rangle$ and $\left|\Gamma_{4}++\right\rangle$ corresponding to the same $m_{J}$ are mixed by the effective electron-hole exchange interaction. For each $m_{J}$ the appropriate effective mass cquation is the $3 \times 3$ matrix which acts on the envelope components $b_{+-} \Phi^{+-}$, $l_{-+} \Phi^{-+}$and $b_{--} \Phi^{--}$, where for the normalized $\Phi^{\bar{\mu} \bar{\nu}}$ the coefficients $b_{\bar{\mu} \bar{\nu}}$ are expected to be about $\left|b_{+-}\right| \approx 1$ and $\left|b_{-+}\right| \ll 1,\left|b_{--}\right| \ll 1$. 


\section{Numerical results and discussion}

We take the simple model of the band energies for the conduction and valence bands in the vicinity of $k=0$ :

and

$$
E_{\mathrm{c}}(k)=E_{\mathrm{G}}+\frac{\hbar^{2} k^{2}}{2 m_{\mathrm{e}}}
$$

$$
E_{v}(k)=-\frac{\hbar^{2} k^{2}}{2 m_{\mathrm{h}}} \text {. }
$$

It is not possible to solve the four-body problem exactly and we therefore employ a variational technique. We confine the problem to the case of the total momentum of the biexciton being equal to zero, $K=0$. We multiply the left hand side of Eq. (32) by $\Phi^{++}$and by $\Phi^{--}$and integrate over the coordinates of particles. From the two non-trivial solutions of the obtained secular equation we choose for the ground state the lower energy $E_{(\text {biex })}$

where

$$
\begin{aligned}
E_{(\mathrm{biex})}=\frac{1}{2}\{ & E_{0}^{++}+\Delta^{++}+E_{0}^{--}+\Delta^{--} \\
& \left.-\left[\left(E_{0}^{++}+\Delta^{++}-E_{0}^{--}-\Delta^{--}\right)^{2}+4 \Delta^{2}\right]^{1 / 2}\right\},
\end{aligned}
$$

$$
\begin{aligned}
E_{0}^{\bar{\mu} \bar{\nu}} & =\left\langle\Phi^{\overline{\mu \nu}}\left|H_{0}\right| \Phi^{\bar{\mu} \bar{\nu}}\right\rangle, \\
\Delta^{\bar{\mu} \bar{\nu}} & =\mathcal{Z}_{\bar{\mu} \bar{\nu}} \tilde{J}\left\langle\Phi^{\bar{\mu}}\left|\delta_{+}\right| \Phi^{\bar{\mu} \bar{\nu}}\right\rangle \quad \text { with } \quad \mathcal{Z}_{++}=\frac{1}{2} \text { and } \mathcal{Z}_{--}=\frac{1}{6}, \\
\Delta & =\frac{1}{2 \sqrt{3}} \tilde{J}\left\langle\Phi^{--}\left|\delta_{-}\right| \Phi^{++}\right\rangle .
\end{aligned}
$$

The value of the exchange integral $\tilde{J}$ (for transverse excitons) is taken from the measured free exciton $\Gamma_{5}-\Gamma_{2}$ splitting $\Delta_{\text {exch }}^{\text {ex }}=6.2 \mathrm{meV}$, where $\Delta_{\text {exch }}^{\text {ex }}=$ $(2 / 3) \tilde{J}|F(0)|^{2} \Omega[8]$ with $F(0)$ - the value of the exciton envelope at zero electron-hole distance $|F(0)|^{2}=1 / \pi a_{\mathrm{ex}}^{3}\left(a_{\mathrm{ex}}=\epsilon \hbar^{2} / \mu e^{2}\right.$ is the free exciton Bohr radius, $\mu$ is the reduced mass). The energy $E_{(\text {biex })}$, expressed with the parameters of the trial wave functions $\Phi^{++}$and $\Phi^{--}$, is numerically minimized. The form of $\Phi^{++}$is the one used by Brinkman et al. [4]

$$
\Phi^{++}=[\psi(r) / S(R)] f(R),
$$

where $\psi$ is a function only of the electron-hole distances

$$
\psi=2 \exp \left[-\left(s_{1}+s_{2}\right) / 2\right] \cosh \left[\beta\left(t_{1}-t_{2}\right) / 2\right]
$$

with $s_{1}=k\left(r_{12}+r_{14}\right), s_{2}=k\left(r_{32}+r_{34}\right), t_{1}=k\left(r_{12}-r_{14}\right), t_{2}=k\left(r_{32}-r_{34}\right)$ and $S^{2}(R)=\int \psi^{2}(r) \mathrm{d}^{3} r_{1} \mathrm{~d}^{3} r_{3}$, while

$$
f(R)=(R / A)^{n} \exp (-R / A)+C \exp (-B R / A)
$$

with $R$ - the hole-hole separation. In the antisymmetric function $\Phi^{--} \psi$ is taken as

and

$$
\psi=2 \exp \left[-\left(s_{1}+s_{2}\right) / 2\right] \sinh \left[\beta\left(t_{1}-t_{2}\right) / 2\right]
$$

$$
f(R)=R\left[(R / A)^{n} \exp (-R / A)+C \exp (-B R / A)\right] .
$$


TABLE I

Parameters obtained for the electron-hole mass ratio $\sigma=m_{\mathrm{e}} / m_{\mathrm{h}}=0.02$.

\begin{tabular}{l|c|c|c|c|c|c}
\hline \hline parameter & $n$ & $k$ & $\beta$ & $A$ & $B$ & $C$ \\
\hline symmetric function $\Phi^{++}$ & 6 & 1.14 & 0.76 & 0.24 & 0.18 & 1.9 \\
antisymmetric function $\Phi^{--}$ & 5 & 1.33 & 1.03 & 0.28 & 0.48 & 4.8
\end{tabular}

The variational parameters $n$ (integer), $k, \beta, A, B, C$ of the functions $\Phi^{++}$and the corresponding parameters of $\Phi^{--}$are varied independently in the minimization procedure.

The values of parameters used in the calculation of the ground state energy $E_{(\text {biex })}$ are listed in Table I.

The binding energy of an biexciton is defined in accordance with Eqs. (33), $(36)$, and (38) as

$$
E_{\mathrm{B}}=-E_{(\text {biex })}+\left(2 E_{\mathrm{G}}+E_{0}\right)-2 R_{x},
$$

where $E_{G}$ is the gap energy, $E_{0}$ is the ground state energy of the electrons filling the valence band and $R_{x}$ is the free exciton rydberg $R_{x}=\mu e^{4} / 2 \epsilon^{2} \hbar^{2}$. In $\mathrm{CuCl}$ $R_{x}=190 \mathrm{meV}$. The biexciton binding energies $E_{\mathrm{B}}$ observed and calculated are listed in Table II. For the ratio of the linear coefficients $b_{--} / b_{++}$, which is a measure of the admixture of the antisymmetric envelope function to the symmetric one, we have got $b_{--} / b_{++}=0.052$.

TABLE II

Biexciton binding energies in $\mathrm{CuCl}$. All energies are in $\mathrm{meV}$.

\begin{tabular}{l|c}
\hline \hline observed [11-13] & 44 \\
\hline $\begin{array}{l}\text { Theoretical (obtained from minimization of } E_{(\text {biex })}=E_{0}^{++}, \text {Eq. (39), } \\
\text { with symmetric envelope function, without exchange correction) }\end{array}$ & 36 \\
\hline $\begin{array}{l}\text { Theoretical (obtained from minimization of } E_{(\text {biex })}=E_{0}^{++}+\Delta^{++} \\
\text {Eq. (39), with symmetric envelope and with exchange term) }\end{array}$ & 24 \\
\hline Present result & 26
\end{tabular}

Some of previous calculations of the biexciton binding (dissociation) energy $E_{\mathrm{B}}$ in $\mathrm{CuCl}$, based on the Hamiltonian without electron-hole exchange terms, are in excellent agreement with experimental data, for example that $44 \mathrm{meV}$ deduced from Wehner's theorem [14]. However, the electron-hole exchange interaction taken into consideration in calculations reduces $E_{\mathrm{B}}$ significantly, as it was shown by Forney et al. [9] and also in our present calculations (see Table II). The difference between $E_{\mathrm{B}}=24 \mathrm{meV}$ and $E_{\mathrm{B}}=26 \mathrm{meV}$ listed in Table II is due to the admixture (i.e. the ratio $b_{--} / b_{++}=0.052$ ) of the antisymmetric envelope function to the symmetric one.

In the simple band model, which was formerly considered by the author $[1,2]$ and by Hayashi [3], the biexciton binding energy obtained with the trial wave function (40) is $E_{\mathrm{B}}=28 \mathrm{meV}$.

We expect (by analogy to the calculations performed for an exciton bound 
to a neutral donor [6]) that the relative corrections due to the admixture of antisymmetric envelope function are more significant for the oscillator-strength of biexciton-exciton transitions than for the biexciton binding energy.

We note that in the case of $\mathrm{CdS}$ and $\mathrm{ZnS}$ (crystals of wurtzite structure) the ratio $b_{--} / b_{++}$is of about $20 \%$ [2].

\section{Acknowledgments}

I am very grateful to Prof. M. Suffczyniski and Dr. P. Janiszewski for many stimulating discussions.

\section{Appendix}

In the $\mathrm{CuCl}$ crystal the valence Bloch functions $\psi_{v, k}$ liave no separated spin dependent part. Using the linearity of $W(i, j)$ we may expand $W(i, j)$ of Eq. (12) in the form

$$
\begin{aligned}
& W(i, j)=\sum_{l, k} \sum_{l^{\prime}, k^{\prime}} \\
& \left|\psi_{c, l}\right\rangle\left|K \psi_{v, k}\right\rangle\left\langle\psi_{c, l}\left|\left\langle K \psi_{v, k}|W(i, j)| \psi_{c, l^{\prime}}\right\rangle\right| K \psi_{v, k^{\prime}}\right\rangle\left\langle\psi_{c, l^{\prime}}\right|\left\langle K \psi_{v, k^{\prime}}\right|,
\end{aligned}
$$

where in the two-band model we have the completeness relation

$$
\sum_{l, k}\left|\psi_{c, l}\right\rangle\left|K \psi_{v, k}\right\rangle\left\langle\psi_{c, l}\right|\left\langle K \psi_{v, k}\right|=1
$$

Since the biexciton states are constructed from the Bloch functions with the wave vectors, whose range (in the vicinity of $k=0$ ) is small compared to the dimension of the Brillouin zone, we evaluate the periodic parts $u_{n, k}$ (it has been assumed that $u_{n, k}$ varies slowly with $k$ over the range of interest) in (A1) and (A2) with $c_{m_{i}}(i)$ and $\left.v_{m_{j}}(j)\right)$ defined in Eqs. (1-2):

$$
\left|\psi_{c, l}\right\rangle\left|K \psi_{v, k}\right\rangle \approx\left|\mathrm{e}^{\mathrm{i} \boldsymbol{l} \cdot \boldsymbol{r}_{\mathrm{i}}} c_{m_{i}}(i)\right\rangle\left|\mathrm{e}^{-\mathrm{i} \boldsymbol{k} \cdot \boldsymbol{r}_{j}} v_{m_{j}}(j)\right\rangle=\left|l, m_{i} ;-k, m_{j}\right\rangle \text {. }
$$

Using Eqs. (12-14), (A1) and (1-2) we expand $W(i, j)$ as follows:

$$
\begin{aligned}
& W(i, j)=\sum_{l, k} \sum_{m_{i}, m_{j}} \sum_{l^{\prime}, k^{\prime}} \sum_{m_{i}^{\prime}, m_{j}^{\prime}} \\
& \quad\left|l, m_{i} ;-k, m_{j}\right\rangle\left\langle l, m_{i} ;-k, m_{j}|W(i, j)| l^{\prime}, m_{i}^{\prime} ;-k^{\prime}, m_{j}^{\prime}\right\rangle\left\langle l^{\prime}, m_{i}^{\prime} ;-k^{\prime}, m_{j}^{\prime}\right| \\
& =\sum_{l, k} \sum_{l^{\prime}, k^{\prime}}\left(\sqrt{\frac{2}{3}}\left|l, \frac{1}{2} ;-k, \frac{1}{2}\right\rangle\left\langle 1\left|-\sqrt{\frac{1}{3}}\right| l, \frac{1}{2} ;-k,-\frac{1}{2}\right\rangle\langle 0|\right. \\
& \left.-\sqrt{\frac{1}{3}}\left|l,-\frac{1}{2} ;-k, \frac{1}{2}\right\rangle\left\langle 0\left|-\sqrt{\frac{2}{3}}\right| l,-\frac{1}{2} ;-k,-\frac{1}{2}\right\rangle\langle-1|\right) \\
& \times V_{r}\left(l,-k ; l^{\prime},-k^{\prime}\right)\left(\sqrt{\frac{2}{3}}|1\rangle\left\langle l^{\prime}, \frac{1}{2} ;-k^{\prime}, \frac{1}{2}\left|-\sqrt{\frac{1}{3}}\right| 0\right\rangle\left\langle l^{\prime}, \frac{1}{2} ;-k^{\prime},-\frac{1}{2}\right|\right. \\
& \left.-\sqrt{\frac{1}{3}}|0\rangle\left\langle l^{\prime},-\frac{1}{2} ;-k^{\prime}, \frac{1}{2}\left|-\sqrt{\frac{2}{3}}\right|-1\right\rangle\left\langle l^{\prime},-\frac{1}{2} ;-k^{\prime},-\frac{1}{2}\right|\right),
\end{aligned}
$$


where $\left|m_{L}=-1,0,1\right\rangle$ stands for $\psi_{m_{L}}-$ the $p$-like functions and

$$
\begin{aligned}
& \left\langle m_{L}\left|V_{r}\left(l,-k ; l^{\prime},-k^{\prime}\right)\right| m_{L}^{\prime}\right\rangle \\
& =\left\langle\mathrm{e}^{\mathrm{i} l \cdot r_{i}} \phi_{c}\left(r_{i}\right)\left|\left\langle\mathrm{e}^{-\mathrm{i} k \cdot r_{j}} \psi_{m_{L}}\left(r_{j}\right)\left|V\left(r_{i}, r_{j}\right)\right| \mathrm{e}^{\mathrm{i} l^{\prime} \cdot r_{i}} \phi_{c}\left(r_{i}\right)\right\rangle\right| \mathrm{e}^{-\mathrm{i} k^{\prime} \cdot r_{j}} \psi_{m_{L}^{\prime}}\left(r_{j}\right)\right\rangle \\
& =\iint \mathrm{d}^{3} r_{i} \mathrm{~d}^{3} r_{j}\left(\mathrm{e}^{\mathrm{i} l \cdot r_{i}} \phi_{c}\left(r_{i}\right) \mathrm{e}^{-\mathrm{i} k \cdot r_{i}} \psi_{m_{L}}\left(r_{i}\right)\right)^{*} \\
& \quad \times \frac{\mathrm{e}^{2}}{\left|r_{i}-r_{j}\right|}\left(\mathrm{e}^{\mathrm{i} l^{\prime} \cdot r_{j}} \phi_{c}\left(r_{j}\right) \mathrm{e}^{-\mathrm{i} k^{\prime} \cdot r_{j}} \psi_{m_{L}^{\prime}}\left(r_{j}\right)\right) .
\end{aligned}
$$

We assume the matrix element (A5) to be equal zero for $m_{L} \neq m_{L}^{\prime}$. From the symmetry of the crystal it follows that the matrix element (A5) for $m_{L}=m_{L}^{\prime}$ does not depend on $m_{L}$ and has the same value for $m_{L}=0, \pm 1$ [15]

$$
\begin{gathered}
\left\langle m_{L}\left|V_{r}\left(l,-k ; l^{\prime},-k^{\prime}\right)\right| m_{L}^{\prime}\right\rangle=\left\langle 0\left|V_{r}\left(l,-k ; l^{\prime},-k^{\prime}\right)\right| 0\right\rangle \delta_{m_{L} m_{L}^{\prime}} \\
=\frac{1}{N} \tilde{J}(l-k) \delta_{l-k, l^{\prime}-k^{\prime}} \delta_{m_{L} m_{L}^{\prime}},
\end{gathered}
$$

where the exchange integral $\tilde{J}(\kappa)$ for $\kappa=l-k=l^{\prime}-k^{\prime}$ can be expanded in terms of Wannier functions $a_{n}(r-R)$ [16] (the factor $1 / N$ arises from the normalization of the Bloch functions, Eq. (3), and the periodicity of their $u$-parts)

$$
\begin{aligned}
\tilde{J}(\kappa)= & \iint \mathrm{d}^{3} r_{1} \mathrm{~d}^{3} r_{2} a_{c}^{*}\left(r_{1}\right) a_{v}^{*}\left(r_{2}\right) \frac{e^{2}}{r_{12}} a_{v}\left(r_{1}\right) a_{c}\left(r_{2}\right) \\
& +\frac{4 \pi}{3} \frac{1}{\Omega} \frac{3\left(\mu_{v c} \cdot \kappa\right)\left(\mu_{c v} \cdot \kappa\right)-\left(\mu_{c v} \cdot \mu_{v c}\right) \kappa^{2}}{\kappa^{2}}+\mathcal{O}\left(\kappa^{2}\right)
\end{aligned}
$$

with the transition dipole moment $\mu_{v c}=\int a_{v}^{*}(r) e r a_{c}(r) \mathrm{d}^{3} r$. For transverse excitons $\left(\boldsymbol{\kappa} \cdot \boldsymbol{\mu}_{c v}=0\right)(\mathrm{A} 7)$ simplifies to

$$
\tilde{J}=\iint \mathrm{d}^{3} r_{1} \mathrm{~d}^{3} r_{2} a_{c}^{*}\left(r_{1}\right) a_{v}^{*}\left(r_{2}\right) \frac{e^{2}}{r_{12}} a_{v}\left(r_{1}\right) a_{c}\left(r_{2}\right)-\frac{4 \pi}{3} \frac{\left|\mu_{v c}\right|^{2}}{\Omega} .
$$

From Eqs. (A4-A7) we get

$$
\begin{aligned}
& W(i, j)= \sum_{l, k} \sum_{l^{\prime}, k^{\prime}} \frac{1}{3 N} \tilde{J}(l-k) \delta_{l-k, l^{\prime}-k^{\prime}}\left[2\left|l, \frac{1}{2} ;-k, \frac{1}{2}\right\rangle\left\langle l^{\prime}, \frac{1}{2} ;-k^{\prime}, \frac{1}{2}\right|\right. \\
&+2\left|l,-\frac{1}{2} ;-k,-\frac{1}{2}\right\rangle\left\langle l^{\prime},-\frac{1}{2} ;-k^{\prime},-\frac{1}{2}\left|+\left(\left|l, \frac{1}{2} ;-k,-\frac{1}{2}\right\rangle+\left|l,-\frac{1}{2} ;-k, \frac{1}{2}\right\rangle\right)\right.\right. \\
&\left.\quad \times\left(\left\langle l^{\prime}, \frac{1}{2} ;-k^{\prime},-\frac{1}{2}\right|+\left\langle l^{\prime},-\frac{1}{2} ;-k^{\prime}, \frac{1}{2}\right|\right)\right] .
\end{aligned}
$$

Since the basis four-particle states |\rangle , Eq. (23), are antisymmetric under the permutation of two electrons $(i=1,3)$ or two holes $(j=2,4)$ the matrix elements of $W(i, j)$ taken between these states |\rangle satisfy the equations $\langle|W(1,2)|\rangle^{\prime}=$ $\langle|W(1,1)|\rangle^{\prime}=\langle|W(3,2)|\rangle^{\prime}=\langle|W(3,4)|\rangle^{\prime}$. Thus we may consider the term $\langle|W(1,2)|\rangle^{\prime}$ only. Taking the Fourier transform of the matrix element (29) we get for the tcrm due to $W(1,2)$ the following expression: 


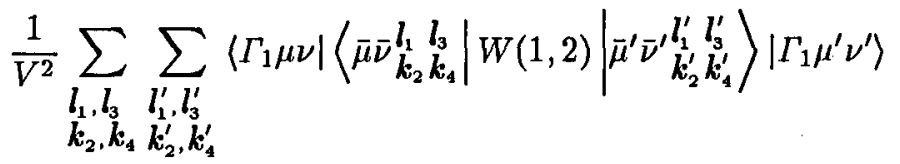

$$
\begin{aligned}
& \times B_{K}^{\bar{\mu}^{\prime} \bar{\nu}^{\prime}}\left(\begin{array}{l}
l_{1}^{\prime} l_{3}^{\prime} \\
k_{2}^{\prime} \\
k_{4}^{\prime}
\end{array}\right) \exp \left[\mathrm{i}\left(l_{1} \cdot r_{1}+l_{3} \cdot r_{3}-k_{2} \cdot r_{2}-k_{4} \cdot r_{4}\right)\right] \\
& =4 Z\left(\begin{array}{l}
\bar{\mu}, \bar{\nu} \\
\bar{\mu}^{\prime} \bar{\nu}^{\prime}
\end{array}\right) S^{\bar{\mu} \bar{\nu}}\left(\begin{array}{l}
r_{1} r_{3} \\
r_{2} r_{4}
\end{array}\right) \frac{1}{V^{2}} \sum_{\substack{l_{1}, l_{3} \\
k_{2}, k_{4}}} \sum_{\substack{l_{1}^{\prime}, l_{3}^{\prime} \\
k_{2}^{\prime}, k_{4}^{\prime}}} \frac{1}{N} \tilde{J}\left(l_{1}-k_{2}\right) \delta_{l_{1}-k_{2}, l_{1}^{\prime}-k_{2}^{\prime}} \delta_{l_{3}, l_{3}} \delta_{k_{4}, k_{4}^{\prime}} \\
& \times B_{\boldsymbol{K}}^{\bar{\mu}^{\prime} \bar{\nu}^{\prime}}\left(\begin{array}{l}
l_{1}^{\prime} l_{3}^{\prime} \\
k_{2}^{\prime} \\
k_{4}^{\prime}
\end{array}\right) \exp \left[\mathrm{i}\left(l_{1} \cdot r_{1}+l_{3} \cdot r_{3}-k_{2} \cdot r_{2}-k_{4} \cdot r_{4}\right)\right] \text {, }
\end{aligned}
$$

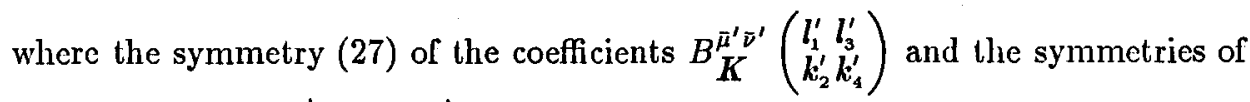

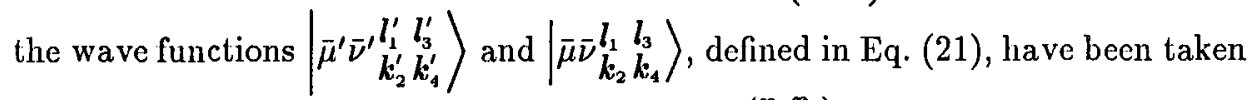
into account. The symmetrization operator $S^{\bar{\mu} \bar{\nu}}\left(\begin{array}{l}\boldsymbol{r}_{1} \boldsymbol{r}_{2} \\ \boldsymbol{r}_{2} \boldsymbol{r}_{4}\end{array}\right)$ is defined by the formula

$$
\begin{aligned}
& S^{\bar{\mu} \bar{\nu}}\left(\begin{array}{c}
r_{1} \\
r_{2} \boldsymbol{r}_{3}
\end{array}\right) f\left(\boldsymbol{r}_{1}, r_{3}, \boldsymbol{r}_{2}, \boldsymbol{r}_{4}\right)=\frac{1}{4}\left[f\left(\boldsymbol{r}_{1}, \boldsymbol{r}_{3}, \boldsymbol{r}_{2}, \boldsymbol{r}_{4}\right)\right. \\
& \left.+\bar{\mu} f\left(\boldsymbol{r}_{3}, \boldsymbol{r}_{1}, \boldsymbol{r}_{2}, \boldsymbol{r}_{4}\right)+\bar{\nu} f\left(\boldsymbol{r}_{1}, \boldsymbol{r}_{3}, \boldsymbol{r}_{4}, \boldsymbol{r}_{2}\right)+\bar{\mu} \bar{\nu} f\left(\boldsymbol{r}_{3}, \boldsymbol{r}_{1}, \boldsymbol{r}_{4}, \boldsymbol{r}_{2}\right)\right] .
\end{aligned}
$$

The coefficients $Z\left(\begin{array}{l}\bar{\mu} \\ \bar{\mu}^{\prime}, \bar{\nu}^{\prime}\end{array}\right)$ arise immediately from the formula (A9) of the $W(1,2)$ operator and from the relations $(28), Z\left(\begin{array}{c}++ \\ +\end{array}\right)=1 / 2, Z\left({ }_{-+}^{+}\right)=Z\left(\begin{array}{l}- \\ +\end{array}\right)=-1 /(2 \sqrt{3})$, $Z(\overline{--})=1 / 6$.

Assuming the longitudinal-transverse splitting in a free exciton as small compared to $\tilde{J}$, Eq. (A8), and approximating the exchange integral in Eq. (A10) by (A8) we get

$$
\begin{aligned}
& 4 Z\left(\begin{array}{l}
\bar{\mu} \\
\bar{\mu}, \bar{\nu}^{\prime}
\end{array}\right) S^{\bar{\mu} \bar{\nu}}\left(\begin{array}{l}
\boldsymbol{r}_{1} \boldsymbol{r}_{3} \\
\boldsymbol{r}_{2} \boldsymbol{r}_{4}
\end{array}\right) \frac{1}{V^{2}} \sum_{\substack{l_{1}, l_{3} \\
k_{2}, k_{4}}} \sum_{\chi} \frac{1}{N} \tilde{J}\left(l_{1}-k_{2}\right) B_{K}^{\bar{\mu}^{\prime} \bar{\nu}^{\prime}}\left(\begin{array}{l}
l_{1}+\chi l_{3} \\
k_{2}+\chi k_{1}
\end{array}\right) \\
& \times \exp \left[\mathrm{i}\left(\boldsymbol{l}_{1} \cdot \boldsymbol{r}_{1}+\boldsymbol{l}_{3} \cdot \boldsymbol{r}_{3}-k_{2} \cdot \boldsymbol{r}_{2}-k_{4} \cdot \boldsymbol{r}_{4}\right)\right]=4 Z\left(\begin{array}{l}
\bar{\mu} \bar{\nu} \\
\bar{\mu}^{\prime} \bar{\nu}^{\prime}
\end{array}\right) \tilde{J} S^{\bar{\mu} \bar{\nu}}\left(\begin{array}{l}
r_{1} \boldsymbol{r}_{3} \\
\boldsymbol{r}_{2} \boldsymbol{r}_{4}
\end{array}\right) \\
& \times \frac{1}{V^{2}} \sum_{\substack{\lambda_{1}, l_{3} \\
\boldsymbol{\kappa}_{2}, k_{4}}} B_{K}^{\bar{\mu}^{\prime} \bar{\nu}^{\prime}}\left(\begin{array}{l}
\lambda_{1} l_{3} \\
\boldsymbol{\kappa}_{2} k_{4}
\end{array}\right) \exp \left[\mathrm{i}\left(\lambda_{1} \cdot r_{1}+l_{3} \cdot r_{3}-\boldsymbol{\kappa}_{2} \cdot r_{2}-\boldsymbol{k}_{4} \cdot \boldsymbol{r}_{4}\right)\right] \\
& \times \frac{1}{N} \sum_{\chi} \exp \left[\mathrm{i} \chi \cdot\left(r_{2}-r_{1}\right)\right]=4 Z\left(\begin{array}{l}
\left.\bar{\mu} \overline{\bar{\mu}} \overline{\bar{\nu}^{\prime}}\right) \\
\bar{X}
\end{array}\right) \tilde{J} S^{\bar{\mu} \bar{\nu}}\left(\begin{array}{l}
r_{1} r_{3} \\
r_{2} r_{4}
\end{array}\right)
\end{aligned}
$$

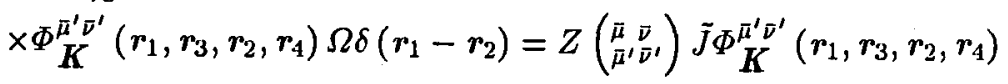

$$
\begin{aligned}
& \times \Omega\left[\delta\left(r_{1}-r_{2}\right)+\bar{\mu} \bar{\mu}^{\prime} \delta\left(r_{3}-r_{2}\right)+\bar{\nu} \bar{\nu}^{\prime} \delta\left(r_{1}-r_{4}\right)+\bar{\mu} \bar{\nu} \bar{\mu}^{\prime} \bar{\nu}^{\prime} \delta\left(r_{3}-r_{4}\right)\right],
\end{aligned}
$$

where the substitutions $\lambda_{1}=l_{1}+\chi, \kappa_{2}=k_{2}+\chi$ and $\chi=k_{2}^{\prime}-k_{2}$, as wcll as, the symmetry relations (31) have been used. 
The Fourier transforms of the one-particle kinetic energy terms and of the Coulomb terms in (29) are obtained with the same procedure used for the exciton [10]. We get, for example, for the one-electron term $H_{\mathrm{IIF}}(1)$

$$
\begin{aligned}
& 4 \delta_{\mu \mu^{\prime}} \delta_{\nu \nu^{\prime}} S^{\bar{\mu} \bar{\nu}}\left(\begin{array}{c}
r_{2} \\
r_{2} \\
r_{4}
\end{array}\right) \frac{1}{r^{2}} \sum_{l_{1}, l_{3}} E_{c}\left(l_{1}\right) \\
& k_{2}, k_{4} \\
& \times B_{K}^{\bar{\mu}^{\prime} \bar{\nu}^{\prime}}\left(\begin{array}{c}
l_{1} \\
l_{3} \\
k_{2}
\end{array}\right) \exp \left[\mathrm{i}\left(l_{1} \cdot r_{1}+l_{3} \cdot r_{3}-k_{2} \cdot r_{2}-k_{4} \cdot r_{4}\right)\right] \\
& =4 \delta_{\mu \mu^{\prime}} \delta_{\nu \nu^{\prime}} S^{\bar{\mu} \bar{\nu}}\left(\begin{array}{c}
r_{1} \\
r_{2} \\
r_{3}
\end{array}\right) E_{c}\left(-\mathrm{i} \nabla_{1}\right) \Phi_{K}^{\bar{\mu}^{\prime} \bar{\nu}^{\prime}}\left(r_{1}, r_{3}, r_{2}, r_{4}\right) \\
& =2 \delta_{\mu \mu^{\prime}} \delta_{\nu \nu^{\prime}}\left[E_{c}\left(-\mathrm{i} \nabla_{1}\right)+E_{c}\left(-\mathrm{i} \nabla_{3}\right)\right] \Phi_{K}^{\bar{\mu}^{\prime} \bar{\nu}^{\prime}}\left(r_{1}, r_{3}, r_{2}, r_{4}\right)
\end{aligned}
$$

and, for example, for the electron-hole Coulomb term $e^{2} / \epsilon r_{12}$

$$
\begin{aligned}
& 4 \delta_{\mu \mu^{\prime}} \delta_{\nu \nu^{\prime}} S^{\bar{\mu} \bar{\nu}}\left(\begin{array}{l}
r_{1} r_{3} \\
r_{2} r_{4}
\end{array}\right) \frac{1}{V^{2}} \sum_{\substack{l_{1}, l_{3} \\
k_{2}, k_{4}}} \frac{1}{V} \sum_{q} U(q) \\
& \times B_{K}^{\bar{\mu}^{\prime} \bar{\nu}^{\prime}}\left(\begin{array}{l}
l_{1}-q l_{3} \\
k_{2}-q k_{4}
\end{array}\right) \exp \left[i\left(l_{1} \cdot r_{1}+l_{3} \cdot r_{3}-k_{2} \cdot r_{2}-k_{4} \cdot r_{4}\right)\right] \\
& =4 \delta_{\mu \mu^{\prime}} \delta_{\nu \nu^{\prime}} S^{\bar{\mu} \bar{\nu}}\left(\begin{array}{l}
\boldsymbol{r}_{1} \\
r_{2} r_{3}
\end{array}\right) \frac{e^{2}}{\epsilon r_{12}} \Phi_{K}^{\bar{\mu}^{\prime} \bar{\nu}^{\prime}}\left(r_{1}, r_{3}, r_{2}, r_{4}\right) \\
& =\delta_{\mu \mu^{\prime}} \delta_{\nu \nu^{\prime}} \frac{e^{2}}{\epsilon}\left[\frac{1}{r_{12}}+\frac{1}{r_{14}}+\frac{1}{r_{32}}+\frac{1}{r_{34}}\right] \Phi_{K}^{\bar{\mu}^{\prime} \bar{\nu}^{\prime}}\left(r_{1}, r_{3}, r_{2}, r_{4}\right),
\end{aligned}
$$

where $U(q)$ is the Fourier transform of the electron-hole interaction potential, $U(q)=\left(e^{2} / \epsilon\right) \int \mathrm{d}^{3} r \exp (\mathrm{i} q \cdot r) / r=4 \pi e^{2} / \epsilon q^{2}$.

As a consequence, the Fourier transform of Eq. (29) becomes Eq. (32).

\section{References}

[1] W. Ungier, Acta Phys. Pol. A 73, 337 (1988).

[2] W. Ungier, Solid State Commun. 69, 53 (1989).

[3] H. Hayashi, Phys. Reu. B 38, 4016 (1988).

[4] W.F. Brinkman, T.M. Rice, B. Bell, Phys. Rev. B 8, 1570 (1973).

[5] W. Ungier, M. Suffczyński, Phys. Rev. B 27, 3656 (1983).

[6] G. Staszewska, M. Suffczyński, W. Ungier, L. Wolniewicz, J. Phys. C 17, 5171 (1984).

[7] K.F. Koster, J.O. Dimmock, R.G. Wheeler, H. Statz, Properties of the Thirty-Two Point Groups, M.I.T. Press, Cambridge (Massachusetts) 1963.

[8] F. Bassani, J.J. Forney, A. Quattropani, Phys. Status Solidi B 65, 591 (1974).

[9] J.J. Forney, A. Quattropani, F. Bassani, Nuovo Cimento B 22, 153 (1974).

[10] J.O. Dimmock, Semiconductors and Semimetals, Eds. R.K. Willardson, A.C. Beer, Vol. 3, Academic Press, New York 1967, p. 259. 
[11] M.A. Lampert, Phys. Rev. Lett. 1, 450 (1958).

[12] J.B. Grun, S. Nikitine, A. Bivas, R. Levy, J. Lumin. 1, 241 (1970).

[13] H. Souma, T. Gota, T. Ohta, M. Ueta, J. Phys. Soc. Jpn. 29, 697 (1970).

[14] R.K. Wehner, Solid Slate Commun. 7, 457 (1969).

[15] B. Arfi, W.T. Masselink, Yia-Chung Clıang, Phys. Rev. B 33, 2401 (1986).

[16] Y. Onodera, Y. Toyozawa, J. Phys. Soc. Jpn. 22, 833 (1967). 